\title{
Prospective Randomized Comparison of Open versus Transperitoneal Laparoscopic Ureterolithotomy: Experience of a Single Center from Northern India
}

\author{
Manish Garg ${ }^{a} \quad$ Vishwajeet Singh ${ }^{a} \quad$ Rahul J. Sinha ${ }^{a} \quad$ Satya N. Sankhwar \\ Manoj Kumar ${ }^{\mathrm{a}} \quad$ Amit Kumar $^{\mathrm{a}} \quad$ Jai Prakash ${ }^{\mathrm{a}}$ Pradeep Kumar ${ }^{\mathrm{a}}$ Mohit Pandey

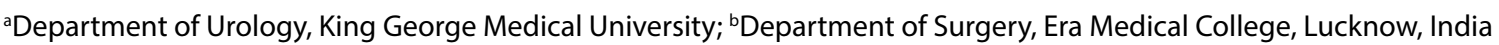

\section{Key Words}

Laparoscopy • Ureterolithotomy • Ureteral calculus

\section{Abstract}

Aim: Prospective randomized study on transperitoneal laparoscopic ureterolithotomy (TPLU) versus open ureterolithotomy (OU) for treatment of large impacted ureteric stones $(\geq 1.5 \mathrm{~cm})$ and assessment of overall results. Material \& Methods: In a prospective study between 2010 to 2012, 30 patients underwent TPLU and 30 OU based on 1: 1 randomization. The operation was indicated primarily in 44 cases or after failed shock-wave lithotripsy/ureteroscopy in 16 cases. Two groups were compared for operative time, success rate, visual pain score, analgesic requirement, hospital stay, and postoperative complications. Statistical analysis was performed with SPSS ${ }^{\circledR}$ version 16.0 using Fisher exact or Mann-Whitney $U$ tests with $p<0.05$ considered statistically significant. Results: The difference in visual pain score $(6.2$ in TPLU group vs 3.1 in OU group on day $1 ; 4.8$ vs. 2.4 on day 2) and tramadol requirements ( $184.32 \mathrm{mg}$ in TPLU group vs. $150.87 \mathrm{mg}$ in OU group on day 1; $97.34 \mathrm{mg}$ vs. $65.56 \mathrm{mg}$ on day 2) were statistically significant and more in OU. Hospital stay and convalescence were significantly lower in the TPLU. However, stone removal in one attempt was similar in both the groups. Conclusion: Although successful stone removal rates are equal in both groups, TPLU is associated significantly with less postoperative pain, less analgesic requirement, shorter hospital stay and short convalescence in comparison to OU.

Copyright $\odot 2013$ S. Karger AG, Basel
\end{abstract}

\section{Introduction}

Currently, extracorporeal shock wave lithotripsy (ESWL) and ureteroscopic stone removal (URS) are the preferred techniques for treatment of ureteric stones with antegrade approach such as percutaneous nephrolithotomy (PNL) may be employed selectively in impacted upper ureteric calculi. Regardless of the status of ESWL, URS or PNL, there are circumstances which decide the best mode of treatment like the stone parameters, characteristics of patient or the surgeon's skill [1]. Laparoscopic or open ureterolithotomy (OU) may be used as primary treatment of large, impacted ureteral stones of more than $1.5 \mathrm{~cm}$ or as a salvage procedure in failed cases of ESWL and attempted URS/PNL [2]. As the laparoscopic techniques are increasingly employed for urological diseases [3], few studies had been conducted comparing results of laparoscopic ureterolithotomy with OU but there is a scarcity of prospective randomized study in the present literature $[4,5]$. Laparoscopic ureterolithotomy by transperitoneal route is a versatile technique for the ureteric stones in all locations $[6,7]$. The advantages of laparoscopic ureterolithotomy using transperitoneal route are large peritoneal space for instrument handling and intra-corporal suturing making procedure comparatively easy [8]. The present study was executed as prospective randomized comparison between open versus transperitoneal laparoscopic ureterolithotomy (TPLU) for the ureteric calculus in all locations which were not amenable
(C) 2013 S. Karger AG, Basel

1015-9770/13/0072-0083\$38.00/0

Accessible online at:

www.karger.com/cur
Manish Garg

Department of Urology, King George Medical University

IN-226003 Lucknow (India)

E-Mail dr_manugarg@yahoo.co.in 
to URS or ESWL/PNL and also used primarily for large impacted $(\geq 1.5 \mathrm{~cm})$ ureteric stones.

\section{Material and Methods}

A total of 60 consecutive patients were enrolled in this prospective study from January 2010 to December 2012. The institutional ethical approval was obtained and it was in accordance with the declaration of Helsinki. All patients had been diagnosed with large ureteric calculi $(\geq 1.5 \mathrm{~cm})$ located in any part of the ureter. Patients were randomized into 1:1 ratio. Of 60 patients, 30 patients were performed OU and 30 TPLU. Patients unwilling for randomization, deranged coagulation profile and associated vertebrospinal deformities were excluded from the study. The procedures were indicated primarily for large, impacted ureteric stones in 44 cases and as salvage procedure in failed cases of URS/ ESWL in 16 patients respectively. Apart from the clinical history and examination, complete hemogram, renal function tests, liver function test, serum calcium, serum uric acid, urine culture and sensitivity, renal and bladder ultrasound, coagulation profiles, $\mathrm{X}$-ray kidney, ureter and bladder (X-ray KUB) were performed in all cases. In patients with impacted stones presented with pain and or renal failure, cystoscopy and double $\mathbf{J}$ (DJ) stenting was performed. Percutaneous nephrostomy was done in cases in which either DJ stent could not be inserted or if there was infected hydroureteronephrosis. The chest X-ray PA view, ECG and pulmonary function test were performed to assess fitness for anesthesia. Urinary tract infection (UTI) was treated considering antibiotic sensitivity. Radiologic investigations included intravenous urogram (IVU) or noncontrast computed tomography KUB. The calculus size was determined by the preoperative KUB film and longest diameter was measured. Informed written consent was obtained prior to surgical intervention.

Procedures were performed by 2 different surgeons. Operating surgeons doing these surgeries are experienced laparosopic surgeons using laparoscopic technique in different urological procedures like nephrectomy, pyelolithotomy, ureterolithotomy, or reconstructive procedures like pyeloplasty for more than a decade with experience of more than 100 cases using either transperitoneal or retroperitoneal route.

Under general anesthesia, cystoscopy and ureteric catheterization was performed. The ureteric catheter was placed just distal to stone in cases of laparoscopic ureterolithotomy. This step was omitted in cases who had prior DJ stenting and in cases of OU. The patients were placed in the lateral decubitus (kidney position) with a bridge at the flank. In open ureterolithotomy for the upper ureteric stones, the patients were placed in the kidney position and for the mid and lower ureteric stone, patients were placed in supine position with a sand bag placed in back to make the operative area oblique and tilted. Standard subcostal or modified Gibson incision was used depending upon the stone location.

\section{TPLU}

Open port $(10 \mathrm{~mm})$ placement for the camera was done, just distal to the umbilicus and lateral to rectus muscle. The carbon dioxide pneumoperitoneum was then created with pressure maintained at $12 \mathrm{mmHg}$. For the left ureteric stones, a 12-mm port in the iliac fossa at the point where spino-umbilical line crosses the mid-clavicular line and an another 5-mm port in mid-clavicular line just below the costal margin were inserted. On the right side, 12 -mm port was placed just below the costal margin in mid-clavicular line and 5-mm port in iliac fossa. The procedure was started with the colonic reflection and identification of the ureter. The identification of the ureter was facilitated either by the presence of DJ stents or artery catheter. The stone was recognized by bulge and confirmed by touching with dissecting forceps. A longitudinal ureterotomy over the stone was given and extracted by combination of traction and pull movement. The stone grasping forcep was used to hold the stone which was removed through 12-mm port site. Intracorporeal interrupted suturing of ureterotomy was performed by 4-0 polygalactin. The reflected colon was replaced and $18 \mathrm{~F}$ soft silastic abdominal tube drain was placed near to suture line. The port sites closure was performed at the end of the procedure.

\section{Postoperative Course and Care}

The patient kept on nasogastric tube and intravenous fluid till the recovery of bowel sounds. Intravenous broad spectrum antibiotic (ceftriaxone) and tramadol on patient demand was administered. Visual pain analog scoring (VAS) was done on first and second postoperative days. X-ray KUB was done to check the position of DJ stent and stone clearance on the second postoperative day. The drainage tube output if less than $30 \mathrm{ml}$ in 24 hours were removed. The patients were discharged following Foley catheter and drainage tube removal. The DJ stent was removed after 4 weeks of operation under intravenous sedation and antibiotic cover. An intravenous urogram was performed at 3 months of follow-up. Subsequent follow-up of patients were at 6 months and then annually. At each visit the apart from the history and clinical examination, serum creatinine, renal and bladder ultrasound were done. Repeat IVU was indicated if there was increasing hydroureteronephrosis or when the patient was symptomatic.

\section{Statistical Analysis}

The results are presented in mean \pm SD (median) and percentages. The normalcy of the data is being tested using Kolgomorove test. The dichotomous/categorical variables are compared by using Chi-square/Fisher exact test whenever applicable. The continuous normally distributed variables are compared by using unpaired t-test and non-normal variables are compared by using Mann-Whitney U test. The p-value $<0.05$ is considered as significant. All the analysis was carried out using SPSS 16.0 version.

\section{Results}

The baseline demographic characteristics of patients are shown in table 1 . Total 60 patients were included in the study. Both groups are comparable with respect to number of patients, age, sex and stone characteristics (table 1). Stones were present in the upper ureter in 32 cases, mid-ureter in 18 and in the lower ureter in 10 patients respectively. The mean stone largest diameter was $2.5 \pm$ $0.69 \mathrm{~cm}$ in OU group and $2.3 \pm 0.78 \mathrm{~cm}$ in TPLU group (table 1). Two patients had bilateral ureteric calculi. One 
patient had a history of nephrectomy for small atrophic kidney and presented with renal failure with midureteric stone in a solitary kidney. DJ stenting was done to relieve obstruction. The mean operating time in OU was $55 \pm$ 9.7 minutes versus $60 \pm 10.3$ minutes in TPLU $(\mathrm{p}=0.06)$ (table 2). The mean blood loss was $60.84 \pm 24.65 \mathrm{ml}$ in OU and $50.67 \pm 18.43 \mathrm{ml}$ in TPLU group (table 2). Complications were recorded and graded using Dindo-modified Clavien classification of surgical complications (table 3). The overall complications were $16.7 \%$ in OU versus $10 \%$ in TPLU group. This difference of complications between 2 groups was not statistically significant $(\mathrm{p}=0.44)$. One patient in TPLU developed transient ileus managed conservatively. Patients in TPLU group required less analgesic in comparison to OU group. Mean visual pain analog score (VAS) on postoperative day 1 was $6.2 \pm 0.76$ in OU versus $3.1 \pm 0.38$ in TPLU and on the second postoperative day mean VAS was $4.8 \pm 0.72$ in OU versus $2.4 \pm 0.49$ for TPLU respectively (table 2). Stone removal /clearance in one attempt was $100 \%$ in both groups. Two open conversions were observed in TPLU group due extensive scarring and non progression. Both these had a history of ESWL. The mean hospital stay was $6.2 \pm 0.65$ days in OU versus $3.1 \pm 0.49$ days in TPLU group (table 2). The median follow-up in present study was 14.7 months in open and 13.3 months in TPLU group respectively. One patient in OU group developed a short segment stricture at initial operated site at 6 months of follow-up which was managed by retrograde balloon dilatation and DJ stenting for 3 months.

\section{Discussion}

ESWL, ureteroscopy and PNL are the preferred modalities for treatment of ureteral stones and offer considerable advantages over OU [9]. Still there are situations when OU is required in patients with complex calculus disease or in presence of anatomic and physiologic anomalies, as according to the Guidelines of the European Association of Urology (EAU-2008) [10]. After the initial description of laparoscopic ureterolithotomy in 1979 by Wickham [11], now with the advancements in technique and experience, laparoscopy has become a highly effective modality in the treatment of complicated ureteral stones. The safety and feasibility of laparoscopic ureterolithotomy has already been demonstrated and it is a reasonable minimally invasive alternative to OU for ureteral stones not amenable to manage with endoscopic techniques [12-14]. Keeley et al. [15] reported the ad-

Open versus Transperitoneal

Laparoscopic Ureterolithotomy vantages of transperitoneal laparoscopic ureterolithotomy in which there is high probability of removing the entire stone burden in one procedure. In an evidence-based review by Skolarikos et al. [16], the highest level of evidence (IIa) was found for laparoscopic ureterolithotomy supporting the laparoscopic approach of stone extraction and mostly recommended (grade B) for large impacted stones or when endoscopic techniques have failed. Due to scarcity of complex and large stones nowadays, there are few studies which actually compared the laproscopic and open techniques of ureterlithotomy in prospective design and most of the previous laparoscopic studies are unrandomized and not actually comparative [17-19].

Laparoscopic ureterolithotomy can either be retroperitoneal or transperitoneal. In present study transperitoneal ureterolithotomy was used in all patients because this is familiar approach, provides the good working space and identification of anatomical landmarks are easier. The main limiting factor with this approach is the intraperitoneal adhesions in previous abdominal surgeries, but the authors did not encounter any major difficulty in operating such patients. Several authors have recommended the transperitoneal route for ureterolithotomy $[1,6,20]$. In present study, mean blood loss was $60.84 \pm 24.65 \mathrm{ml}$ in OU and $50.67 \pm 18.43 \mathrm{ml}$ in TPLU group $(\mathrm{p}=0.07)$. ElFeel et al. [20] reported a mean blood loss of $62 \mathrm{ml}$ and it was $51 \mathrm{ml}$ in a study reported by Kongchareonsombat et al. [21]. These results are comparable to the present study.

Laparoscopic ureterolithotomy by transperitoneal approach has definite advantage over open ureterolithotomy. Traditionally, the OU requires large muscle cutting incision (usually $10 \mathrm{~cm}$ or larger) which is the main cause of pain and thus resulting delayed recovery and long convalescence period [22]. Laparoscopic surgery can be done with 3-4 small $1-1.5 \mathrm{~cm}$ incisions without muscle cutting for port insertion. This marked benefit could be appreciated from significant difference in pain score and postoperative analgesic requirement between 2 groups. Although, small mini incision ureterolithotomy, utilizing muscle splitting rather than muscle cutting approach, has been described in literature which produce equivalent results and less morbidity in comparison to laparoscopic ureterolithotomy [23]. Further randomized controlled prospective studies are required to elucidate the benefits of mini-incision ureterolithotomy.

The main issue with OU (whether traditional or mini-incision) is the site of incision which should be always according to the position of calculus in the ureter. It may sometimes happen that the stone position may 
Table 1. Baseline demographic characteristics in both the groups

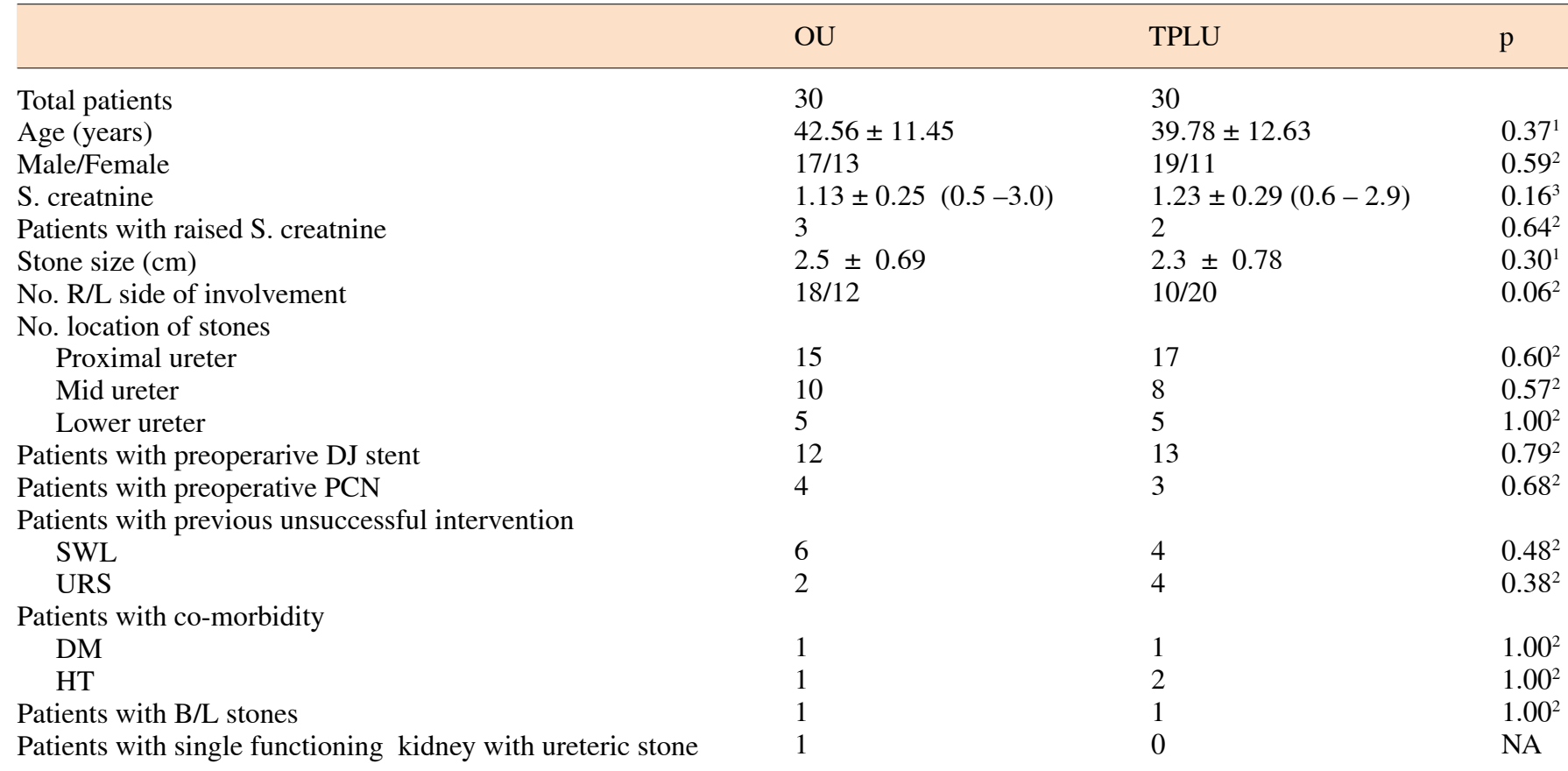

${ }^{1}$ Unpaired t-test; ${ }^{2}$ Chi-square/Fisher exact; ${ }^{3}$ Mann-Whitney U test. NA = Not applicable.

Table 2. Perioperative and postoperative parameters

\begin{tabular}{llll}
\hline & OU & TPLU & p \\
\hline Operative time (minutes) & $55 \pm 9.7$ & $60 \pm 10.3$ & $0.06^{1}$ \\
VAS day 1 & $6.2 \pm 0.76$ & $3.1 \pm 0.38$ & $0.0001^{1}$ \\
VAS day 2 & $4.8 \pm 0.72$ & $2.4 \pm 0.49$ & $0.0001^{1}$ \\
Analgesic tramadol (mg) day 1 & $184.32 \pm 33.45$ & $150.87 \pm 32.87$ & $0.0002^{1}$ \\
Analgesic tramadol (mg) Day 2 & $97.34 \pm 14.40$ & $65.56 \pm 21.35$ & $0.0001^{1}$ \\
Blood loss (ml) & $60.84 \pm 24.65$ & $50.67 \pm 18.43$ & $0.07^{1}$ \\
Days of drain removal & $4.43 \pm 2.44$ & $3.60 \pm 2.85$ & $0.23^{1}$ \\
Hospital stay (days) & $6.2 \pm 0.65$ & $3.1 \pm 0.49$ & $0.0001^{1}$ \\
Follow-up (months) & 14.7 & 3.3 & $0.67^{2}$ \\
Overall complications & $5(16.7 \%)$ & $3(10 \%)$ & $0.44^{3}$ \\
\hline
\end{tabular}

${ }^{1}$ Unpaired t-test; ${ }^{2}$ Mann-Whitney U test; ${ }^{3}$ Chi-square/Fisher exact.

Table 3. Early and late complications by Dindo-modified Clavien Classification of Surgical Complications

\begin{tabular}{|c|c|c|c|c|}
\hline Clavien grading & Complication & OU patients, $n$ & TPLU patients, $\mathrm{n}$ & $\mathrm{p}$ \\
\hline I & transient decrease in renal function & 1 & 0 & NA \\
\hline II & febrile UTI & 1 & 1 & $1.000^{1}$ \\
\hline II & prolonged urine drainage & 1 & 1 & $1.000^{1}$ \\
\hline II & wound infection & 1 & 0 & NA \\
\hline IIIb & stricture formation & 1 & 0 & NA \\
\hline
\end{tabular}

${ }^{1}$ Chi-square/Fisher exact. NA = Not applicable. 
change or up migrate due to dilated ureter before surgery or even intraoperatively. In such cases, it sometimes becomes very difficult to negotiate the calculus though same incision and one has to either make another incision or abandon the procedure for the time being. The transperitoneal laparoscopic route has a definite advantage in avoiding such technical complications as calculus can be easily retrieved with further dissection of the ureter without much increased morbidity. Additional advantages are in cases when there are more than 1 calculi present in different locations of same ureter or in case of bilateral ureteric stones in which both the stones could be retrieved in the same sitting with only slight maneuverability or inserting additional port which is not feasible in the open ureterolithotomy. Also, TPLU may be especially useful when treating patients with concomitant upper urinary tract problems, such as ureteric strictures or uretero-pelvic junction obstruction, which need simultaneous surgical repair.

High success rate depends upon proper patient selection and surgical experience of laparoscopic technique. Historically success rates of transperitoneal uretrolithotomy range from 86 to $100 \%$ [6, 12, 18, 24]. Further studies showed that with increase in experience, overall success rate is above $90 \%$ [25, 26]. Both TPLU and OU have better overall success rates in comparison to presently available other minimally invasive techniques as studied by Basiri et al. [6] who compared URS, PNL and TPLU, and reported stone-free rates of 56, 64, and $88 \%$, respectively. Better overall success rates in a single sitting is the sheer advantage of open or laparoscopic ureterolithotomies over endoscopic techniques $[6,27]$.

In the present study, mean operative time was $60 \pm$ 10.3 minutes in TPLU group and $55 \pm 9.7$ minutes in OU group. El-Feel et al. [20] reported the mean operative time of 145 minutes (range 55 -180 minutes). Skrepetis et al. [4] compared open versus laparoscopic ureterolithotomies in 36 patients and concluded that the operation time was significantly longer in the lapaoscopic group. In contrast, we found operating time comparable in both groups. Our operating time is less in comparison to other mentioned studies because of the technique and expertise of the operating surgeon. Also initial insertion of ureteric catheter or DJ stent reduced the operative time in identification and dissection of the ureter.

Stones were identified by obvious bulge or pinching by Maryland forcep. Upward migration of the stone was prevented by ap plying a laparoscopic Babcock forcep on the ureter above the stone bulge. We made ureterotomy with electrocautery which was easier as compared to the

Open versus Transperitoneal

Laparoscopic Ureterolithotomy incision with a cold knife. Use of diathermy for ureterotomy does not produce adverse effect on ureteric tissue healing [17]. Antegrade DJ stenting was facilitated with help of guidewire [28]. As ureterotomy made was small and exactly over the stone, it was closed with intermittent 2-3 sutures without consuming much time. In a study by Karami et al. [29], stenting the ureter during surgery does not significantly increase the time of surgery and may play important role in prevention of urinary extravasation.

In contrast to most of the published literature on laparoscopic ureterolithotomy, where the success rate in lower ureteric stone is described to be less as compared to middle and upper ureteric calculi [20,30], success rate was equivalent in the present study irrespective of the stone location in the ureter. In lower ureter stone, dissection of the ureter was done with extra caution where the space was less and as the ureter was crossing the iliac vessels, there were more chances of vascular injury. As compared to other locations, the overall procedure time was more for stones which were located in the lower ureter. Abolyosr et al. [31] reported that upper and mid ureteric stones can be safely approached retroperitoneally but for lower ureteric stones transperitoneal approach is a much better option as it gives a better understanding of the anatomical landmarks particularly in the lower part of the ureter.

In the present study, complications were recorded and graded according to Dindo-modified Clavien classification of surgical complications. No major complications occurred during or after the surgery in either of 2 groups, except for 1 patient in the TPLU group who had persistent high drain output due to up-migrated DJ stent, which was repositioned. Temporary ileus was observed in 1 patient in TPLU group and managed conservatively. Temporary ileus is a complication of TPLU also reported by others $[12,20]$. One patient in the open group developed transient derangement of renal function which could be because of infection as this patient had a febrile UTI following the procedure. Overall reported mean complications of laparoscopic transperitoneal urological surgeries range from 14.1 to $19 \%$ [32, 33] and for transperitoneal ureterolithotomy, complications varies from 4 to $18 \%$ in different series [9, 20, 24]. Feyaerts et al. [12] reported overall $8.3 \%$ complications of TPLU. El-Feel et al. [20] reported 4\% and Simforoosh et al. [24] reported $12.2 \%$ of complications, respectively. In an interesting study, Basiri at al. [6] reported $18 \%$ complications in the form of leakage of urine for more than 3 days. Feyaerts et al. [12] reported an average hospital stay of 3.8 
days, El-Feel et al. [20] reported 4.1 days and Basiri et al. [6] reported a mean hospital stay of $5.8 \pm 2.3$ days, respectively. Mean hospital stay in the present study was $3.1 \pm 0.49$ days in TPLU group and $6.2 \pm 0.65$ days in open group, respectively. This allowed early ambulation and early resumption of oral intake in laparoscopic group which was significant as compared to open group. Fang et al. [34] in a study found laparoscopic ureterolithotomy to have higher stone clearance rate and shorter operating time compared with ureteroscopic lithotripsy.

Laparoscopic transperitoneal ureterolithotomy is a safe, minimally invasive and maximally effective treatment modality for skilled surgeons. Difficult situations as previously operated cases, obese patients and lower ureteral stones can be overcome by experience. TPLU can be considered as an intermediate step in gaining experience and developing necessary skills in utilizing laparoscopic approach in more complicated reconstructive or radical urological surgery.

\section{Conclusion}

TPLU is associated with less postoperative pain, less analgesic requirement, shorter hospital stay and early convalescence in comparison to open ureterolithotomy. However, the stone removal rate in one attempt remains equal in both groups irrespective of stone locations.

\section{References}

1 Almeida GL, Heldwein FL, Graziotin TM, Schmitt CS, Teloken C: Prospective trial comparing laparoscopy and open surgery for management of impacted ureteral stones. Actas Urol Esp 2009;33:1108-1114.

-2 Singh V, Sinha RJ, Gupta DK, Kumar M, Akhtar A: Transperitoneal versus retroperitoneal laparoscopies ureterolithotomy: a prospective randomized comparison study. J Urol 2013;189:940-945.

3 Hruza M, Schulze M, Teber D, Gozen AS, Rassweiler JJ: Laparoscopic techniques for removal of renal and ureteral calculi. J Endourol 2009;23:1713-1718.

-4 Skrepetis K, Doumas K, Siafakas I, Lykourinas M: Laparoscopic versus open ureterolithotomy. A comparative study. Eur Urol 2001;40:32-36.

5 Goel A, Hemal AK: Upper and mid-ureteric stones: a prospective unrandomized comparison of retroperitoneoscopic and open ureterolithotomy. BJU Int 2001;88:679-682.

6 6 Basiri A, Simforoosh N, Ziaee A, Shayaninasab H, Moghaddam SM, Zare S: Retrograde, antegrade, and laparoscopic approaches for the management of large, proximal ureteral stones: a randomized clinical trial. J Endourol 2008;22:2677-2680.

7 Mandhani A, Kapoor R: Laparoscopic ureterolithotomy for lower ureteric stones: steps to make it a simple procedure. Indian J Urol 2009;25:140-142.

$>8$ Henkel TO, Rassweiler J, Alken P: Ureteral laparoscopic surgery. Ann Urol (Paris) 1995; 29:61-72.
-9 Preminger GM, Tiselius HG, Assimos DG, Alken P, Buck AC, Gallucci M, Knoll T, Lingeman JE, Nakada SY, Pearle MS, Sarica K, Türk C, Wolf JS Jr: 2007 guideline for the management of ureteral calculi. Eur Urol 2007;52:1610-1631.

10 Tiselius HG, Alken P, Buck AC, Gallucci M, Knoll T, Sarica K, Türk C: EAU Guidelines on Urolithiasis. 2008, European Association of Urology.

11 Wickham JE: The surgical treatment of renal lithiasis; in Wickham JE (ed): Urinary Calculous Disease. Churchill Livingstone, New York, 1979, pp145-198.

-12 Feyaerts A, Rietbergen J, Navarra S, Vallancien G, Guillonneau B: Laparoscopic ureterolithotomy for ureteral calculi. Eur Urol 2001;40:609-613.

13 Flasko T, Holman E, Kovacs G, Tallai B, Toth C, Salah MA: Laparoscopic ureterolithotomy: the method of choice in selected cases. J Laparoendosc Adv Surg Tech A 2005;15: 149-152.

14 Farina Perez LA, Pesqueira Santiago D, Meijide Rico F, Zungri Telo ER: Laparoscopic ureterolithotomy of an iliac ureteral stone forgotten for more than eight years. Actas Urol Esp 2006;30:218-221.

15 Keeley FX, Gialas I, Pillai M, Chrisofos M, Tolley DA: Laparoscopic ureterolithotomy: the Edinburgh experience. BJU Int 1999;84: 765-769.

16 Skolarikos A, Papatsoris AG, Albanis S, Assimos D: Laparoscopic urinary stone surgery: an updated evidence-based review. Urol Res 2010;38:337-344.
17 Gaur DD, Trivedi S, Prabhudesai MR, Madhusudhana HR, Gopichand M: Laparoscopic ureterolithotomy: technical considerations and long-term follow-up. BJU Int 2002;89: 339-343.

-18 Turk I, Deger S, Roigas J, Fahlenkamp D, Schonberger B, Loening SA: Laparoscopic ureterolithotomy. Tech Urol 1998;4:29-34.

19 Hemal AK, Goel A, Kumar M, Gupta NP: Evaluation of laparoscopic retroperitoneal surgery in urinary stone disease. J Endourol 2001;15:701-705.

$\checkmark 20$ El-Feel A, Abouel-Fettouh H, Abdel-Hakim AM: Laparoscopic transperitoneal ureterolithotomy. J Endourol 2007;21:50-54.

-21 Kongchareonsombat W, Atichoksakun S, Kitvikai K, Patcharatrakul S, Chaimuengraj S: Extraperitoneal versus transperitoneal approach of laparoscopy ureterolithotomy in selected patients. J Med Assoc Thai 2010;93: 794-798.

22 Harewood LM, Webb DR, Pope AJ: Laparoscopic ureterolithotomy: the results of an initial series, and an evaluation of its role in the management of ureteric calculi. Br J Urol 1994;74:170-176.

-23 Sharma DM, Maharaj D, Naraynsingh V: Open mini-access ureterolithotomy: the treatment of choice for the refractory ureteric stone? BJU Int 2003;92:614-616.

24 Simforoosh N, Basiri A, Danesh AK, Ziaee SA, Sharifiaghdas F, Tabibi A, Abdi H, Farrokhi F: Laparoscopic management of ureteral calculi: a report of 123 cases. Urol J 2007; 4:138-141. 
-25 El-Moula MG, Abdallah A, El-Anany F, Abdelsalam Y, Abolyosr A, Abdelhameed D, Izaki H, Elhaggagy A, Kanayama HO: Laparoscopic ureterolithotomy: our experience with 74 cases. Int J Urol 2008;15:593-597.

26 Wolf JS: Treatment selection and outcomes: ureteral calculi. Urol Clin North Am 2007;34: 421-430.

$>27$ Ko YH, Kang SG, Park JY, Bae JH, Kang SH, Cho DY, Park HS, Cheon J, Lee JG, Kim JJ: Laparoscopic ureterolithotomy as a primary modality for large proximal ureteral calculi: comparison to rigid ureteroscopic pneumatic lithotripsy. J Laparoendosc Adv Surg Tech A 2011;21:7-13.
28 Mandhani A: A technique of stenting after laparoscopic ureterolithotomy. Indian J Urol 2011;27:418-419.

29 Karami H, Javanmard B, Hasanzadeh-Hadah A, Mazloomfard MM, Lotfi B, Mohamadi R, Yaghoobi M: Is it necessary to place a double J catheter after laparoscopic ureterolithotomy? A four-year experience. J Endourol 2012;26:1183-1186.

30 Matias DB, Alvim RG, Ribas M, de Oliveira BP, Chaves OH: Laparoscopic treatment of ureterolithiasis: our experience. Actas Urol Esp 2009;33:667-669.

31 Abolyosr A: Laparoscopic transperitoneal ureterolithotomy for recurrent lower ureteral stones previously treated with open ureterolithotomy: Initial experience in 11 cases. J Endourol 2007;21:525-529.
32 Lin $\mathrm{YH}$, Chung HJ, Lin AT, Chang YH, Huang WJ, Hsu YS, Chang SC, Chen KK: Complications of pure transperitoneal laparoscopic surgery in urology: the Taipei Veterans General Hospital experience. J Chin Med Asso 2007;70:481-485.

-33 Vallancien G, Cathelineau X, Baumert H, Doublet JD, Guillonneau B: Complications of transperitoneal laparoscopic surgery in urology: review of 1,311 procedures at a single center. J Urology 2002;168:23-26.

34 Fang YQ, Qiu JG, Wang DJ, Zhan HL, Situ J: Comparative study on ureteroscopic lithotripsy and laparoscopy ureterolithotomy for treatment of unilateral upper ureteral stones. Acta Cir Bras 2012;27:266-270. 